\title{
Potential for Energy Recovery of Unpowered Configurations using Power Balance Method Computations
}

\author{
Ngonidzashe.E. Mutangara*, Lelanie. Smith ${ }^{\dagger}$ Kenneth.J. Craig \\ University of Pretoria, Pretoria, South Africa, 0001 \\ Drewan.S. Sanders \\ Cranfield University, Bedford, United Kingdom, MK43 OAL
}

\begin{abstract}
New aircraft developments are made to improve aircraft performance and efficiency. One such method is integrating the propulsion into the airframe. This allows for boundary layer ingestion (BLI) which shows promise of significant power benefits. However, these benefits are difficult to quantify as the propulsion system and aircraft body become meticulously integrated. The thrust and drag are coupled and cannot be defined separately, making conventional performance analysis methods inapplicable. The power balance method (PBM) addresses this by quantifying aircraft performance in terms of mechanical flow power and the change in kinetic energy rate. The primary focus of this work was to perform computational studies implementing the power balance method on unpowered aerodynamic bodies to evaluate their respective drag contributions. A secondary study was also conducted to quantify the energy recovery potential of the various bodies using a Potential for Energy Recovery (PER) factor. The CFD case studies showed that drag obtained using the power balance method agreed to within $2 \%$ of conventional momentum-based approaches. Maximal energy recovery potential was consistently observed at the trailing ends of the geometries, with values ranging between $9-12 \%$.
\end{abstract}

*MEng Student, Department of Mechanical and Aeronautical Engineering; ngonidzashe.e.mutangara@cranfield.ac.uk Student Member AIAA (Corresponding Author).

†Senior Lecturer, Department of Mechanical and Aeronautical Engineering; lelanie.smith@ up.ac.za. Member AIAA.

†Professor, Department of Mechanical and Aeronautical Engineering; ken.craig@up.ac.za.

${ }^{\S}$ Research Fellow, School of Aerospace, Transport and Manufacturing; d.s.sanders@ cranfield.ac.uk Member AIAA. 


\section{Nomenclature}

$$
\begin{aligned}
& A=\text { area } \\
& V \quad=\text { volume } \\
& \rho \quad=\text { fluid density } \\
& D_{F}, L_{F} \quad=\text { body diameter and length } \\
& u, v, w=\text { perturbation velocities } \\
& p, p_{t} \quad=\text { static pressure, total pressure } \\
& \widehat{\boldsymbol{n}}=\text { unit normal vector, out of control volume } \\
& x, y, z=\text { cartesian axis coordinates } \\
& C_{p} \quad=\text { pressure coefficient } \\
& \text { Re } \quad=\text { Reynolds number } \\
& M \quad=\quad \text { Mach Number } \\
& \boldsymbol{V}=\text { fluid velocity }\left(V_{\infty}+u\right) \hat{x}+v \hat{y}+w \hat{z} \\
& V^{2} \quad=\text { fluid speed squared }=\boldsymbol{V} \cdot \boldsymbol{V} \\
& V_{n} \quad=\text { side cylinder normal velocity }\left(v n_{y}+w n_{z}\right) \\
& \overline{\boldsymbol{\tau}} \quad=\text { viscous stress tensor } \\
& F_{x} \quad=\text { net streamwise force in } \mathrm{x} \\
& C D=\text { drag coefficient } \\
& \dot{E}_{a} \quad=\text { axial kinetic energy deposition rate } \\
& \dot{E}_{v} \quad=\text { transverse (vortex) kinetic energy deposition rate } \\
& \dot{E}_{p} \quad=\text { pressure-work deposition rate } \\
& \dot{E}_{w} \quad=\text { lateral wave-outflow energy deposition rate } \\
& P_{S} \quad=\text { net propulsor shaft power } \\
& P_{V} \quad=\text { volumetric mechanical power } \\
& P_{K} \quad=\text { net propulsor mechanical energy inflow rate } \\
& \dot{\varepsilon}=\text { mechanical energy outflow rate } \\
& \Phi \quad=\text { viscous dissipation rate }
\end{aligned}
$$




$$
\begin{array}{ll}
d S & =\text { surface element of the control volume } \\
d V & =\text { volume element of the control volume } \\
W & =\text { aircraft weight } \\
\delta & =\text { boundary layer thickness } \\
\tau & =\text { shear stress } \\
v & =\text { kinematic viscosity }
\end{array}
$$

\section{Subscripts}

()$_{\infty}=$ freestream quantity

()$_{O} \quad=$ quantity on body surface

()$_{\text {ref }}=$ reference quantity

()$_{n f}=$ near-field quantity

()$_{f f} \quad=$ far-field quantity

()$_{\Phi} \quad=$ power balance quantity

()$_{O}^{S C} \quad=$ quantity on side cylinder

()$_{O}^{T P} \quad=$ quantity on transverse plane

()$_{m} \quad=$ recoverable mechanical energy quantity

()$_{T E} \quad=$ trailing edge quantity 


\section{MUTANGARA ET AL.}

\section{Introduction}

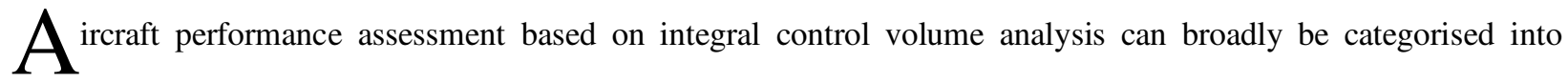
force(momentum), kinetic(mechanical) energy and exergy methods [1]. Momentum-based techniques include nearfield integration, commonly used in most computational fluid dynamics codes, calculating drag as the integration of stresses along the body's physical surfaces. Another is the far-field method which quantifies drag by analyzing flowfield perturbations. Near and farfield methods are based on similar principles (i.e. momentum balance), but their analysis perspectives differ. The near-field approach focuses on the forces experienced by the body as a result of the flow passing over it. In contrast, far-field methods analyze flow disturbances, which occur as a result of the body's presence within a flow field. The near-field approach is limited in that it only allows for drag breakdown into friction and pressure components, whereas far-field methods enable decompositions into more detailed, descriptive and useful constituents. A variety of methods exist [2-7], allowing for aircraft forces to be calculated as a combination of surface (Trefftz plane) and/or volume integrals within the flow, based on expressions obtained from enthalpy and entropy relations.

Despite the advantages of these advanced methods, they are still reliant on thrust definitions for evaluating the efficacy of the propulsion system in terms of power requirements. The division between thrust and drag is a notional concept, and there is no clear inherent way of defining the useful (thrust) work done by the propulsion system [8]. For conventional configurations, there is "sufficient decoupling" between airframe and propulsion system aerodynamics, allowing for thrust definitions that approximate a valid, useful work for performance evaluation. However, where there is tighter integration between airframe and propulsion (as in the case of BLI), the aero-coupling does not allow for a valid distinction of useful work to be obtained from a definition of thrust power. Alternative methods attempt to overcome this challenge by expressing force decompositions within mechanical energy [8,9] and exergy-anergy [10] conservation formulations. The underlying principle is to circumvent thrust-power based performance evaluation, by directly tracing power consumption development of different flow mechanisms within the flowfield. This enables a more holistic treatment of airframe-propulsion system performance evaluations.

Exergy-anergy formulations have shown to be particularly useful in providing complementary aerodynamic characteristic curves yielding a more complete aerodynamic assessment of aircraft configurations $[11,12]$ and classical aerodynamic problems [13-16]. Aerodynamic analyses based on exergy are purely focused on a thermodynamic 
MUTANGARA ET AL.

perspective, offering a clear distinction between reversible and irreversibly lost energy within a flowfield via an analysis of entropy, as shown by Aguirre and Duplaa [16]. Arntz et al. [10,11,17] further show that through its unique decomposition of drag, the recoverable energy within the airframe wake can be quantified. This is done through a figure of merit known as the exergy waste coefficient (EWC), indicating the amount of energy available for utilization by BLI systems. Although opined as a more complete formulation due to its inclusion of first and second law of thermodynamics terms, for typical external aerodynamics applications which neglect heat transfer, exergy formulations effectively simplify to the power balance equation provided by Drela [9]. As energy-based methods focus on a mechanical energy approach, they avoid the use of entropy terms allowing for an improved physical interpretation of the decomposed force terms. Further to this, a factor similar to EWC exists based on mechanical energy analysis known as the Potential for Energy Recovery (PER) introduced by Sanders and Laskaridis [8]. This presents an opportunity to analyze aerodynamic flows from a mechanical energy perspective through these formulations, complementing prior work based on exergy via an alternative method of analysis.

The work in this paper focuses on the numerical implementation of the power balance method on simple benchmark cases for verification within commercial CFD codes. Section II introduces the power balance method, modified via a sequence of simplifications associated with unpowered configurations. Section III presents the case studies analyzed, split between flat plates and aerodynamic bodies, leading to the numerical verification and validation procedure in Section IV. Finally, in Section V, the drag over these geometries is reported using the power balance method and compared against momentum based approaches (i.e. near-field and far-field) for verification.

\section{II.Mathematical Model}

\section{A. Power Balance Method}

\section{Momentum and Force relations}

Aerodynamic analysis using integral control volume methods requires the definition of Control Volumes (CVs) around the aerodynamic body of interest, as shown in Figure 1 . The CV boundary $S$ is partitioned into an inner boundary $S_{B}$ lying on the body surface, and an outer boundary $S_{O}$ encapsulating the flow field. 


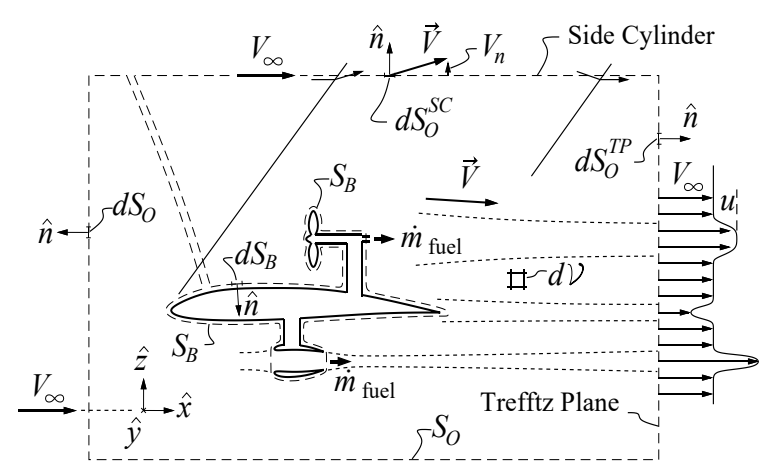

Figure 1 2D cutaway view of 3D Control Volume surrounding an aerodynamic body [9]

The profile drag over the body can be obtained either by computing the near-field on-body force $\boldsymbol{F}_{B}$ or the outerboundary force momentum flow $\boldsymbol{F}_{O}$ via a sequence of integrals over the CV surfaces using equations (1) and (2), provided by Drela [9]. These expressions describe how these forces are evaluated over aerodynamic bodies using near and far-field approaches, respectively.

$$
\begin{gathered}
\boldsymbol{F}_{B}=\oiint[(p \widehat{\boldsymbol{n}}-\overline{\boldsymbol{\tau}})+\boldsymbol{V} \rho \boldsymbol{V} \cdot \widehat{\boldsymbol{n}}] d S_{B} \\
\boldsymbol{F}_{O}=\oiint-\left[\left(p-p_{\infty}\right) \widehat{\boldsymbol{n}}+\left(\boldsymbol{V}-\boldsymbol{V}_{\infty}\right) \rho \boldsymbol{V} \cdot \widehat{\boldsymbol{n}}\right] d S_{O}
\end{gathered}
$$

\section{Mechanical Energy Analysis}

A mechanical energy analysis of the body in Figure 1, provides a prediction of the total flight power required through an estimation of the power outflow from various physical processes. It is, therefore, by the power balance (PB) of the power outflow that the power inflow requirements can be obtained, i.e. $\left(\dot{E}_{\text {inflow }}=\dot{E}_{\text {outflow }}\right)$. Drela [9] expands this to give equation (3) which conveniently separates the power supplying and consuming processes on its left- and righthand sides, respectively.

$$
\underbrace{P_{S}+P_{V}+P_{K}}_{\text {Total power }}=\underset{\text { Total mechanical energy }}{\dot{\varepsilon}_{\text {viscous dissipation }}^{\Phi}}
$$

The method relies on the principle of conservation of mechanical energy, globally over the CV and locally within the wake [18]. The wake energy is defined as the sum of the mechanical energy deposited on the Trefftz plane (TP) and the energy dissipated inside the wake region. This is then equated to the total power, given as the sum of the shaft $\left(P_{S}\right)$, volumetric $\left(P_{V}\right)$ and mechanical inflow power $\left(P_{K}\right)$ from the propulsors. 


\section{MUTANGARA ET AL.}

The total mechanical energy can be broken down further as done in equation (4), shown as the sum of the altitude potential energy, recoverable mechanical energy, and wave energy irreversibly lost to the surroundings.

$$
\underbrace{\dot{\mathcal{E}}}_{\text {Total mechanical energy }}=\underbrace{W \dot{h}}_{\text {altitude potential energy }}+\underbrace{\dot{E}_{a}+\dot{E}_{v}+\dot{E}_{p}}_{\text {recoverable mechanical energy }}+\underbrace{\dot{E}_{w}}_{\text {wave energy }}
$$

\section{Generalised unpowered analysis simplifications}

The analyses covered herein are restricted to unpowered bodies in steady flight; under these conditions, the altitude potential energy and drag power can be related through equation (5). By also considering the shaft and mechanical inflow power in equation (3) to be zero due to the absence of a propulsor, the remaining non-zero terms can be related to the drag power as shown in equation (5). This equation is cast in a force decomposition format to allow a more straightforward evaluation of the body drag. The equation also highlights the recoverable and non-recoverable components of the drag power for unpowered configurations; it is by the utilization of this recoverable energy that BLI obtains its benefits.

$$
\begin{gathered}
\underbrace{D V_{\infty}}_{\text {Drag Power }}=-W \dot{h} \\
=\underbrace{\dot{E}_{a}}_{\text {axial wake energy }}+\underbrace{\dot{E}_{v}}_{\text {transverse wake energy }}+\underbrace{\dot{E}_{p}}_{\text {pressure-work rate }}+\underbrace{\dot{E}_{V}+\Phi}_{\text {irreversibly lost energy }} \\
-\underbrace{P_{\text {mechanical power }}}_{\text {volumetric }}
\end{gathered}
$$

A non-dimensional form of equation (5) can be obtained using the freestream dynamic pressure force power $\left(\frac{1}{2} \rho_{\infty} V_{\infty}^{3} A_{\text {ref }}\right)$. This non-dimensionalisation is directly comparable to the traditional drag coefficient [10], and a depiction of this process is shown in equation (6).

$$
C D=\frac{D}{\frac{1}{2} \rho_{\infty} V_{\infty}^{2} A_{r e f}} ; C \dot{E}_{a}=\frac{\dot{E}_{a}}{\frac{1}{2} \rho_{\infty} V_{\infty}^{3} A_{r e f}}
$$

Lastly, further simplification of the power balance formulation can be done when applied to inviscid flows. For this, viscous dissipation is neglected due to the absence of viscous effects. 


\section{BLI Performance Parameters}

As the power saving coefficient [19] remains a Figure of Merit (FoM) exclusively for powered configurations, Sanders and Laskaridis [8] introduce another FoM known as the Potential for Energy Recovery (PER). This factor evaluates the potential for aerodynamic improvement, similarly done by the EWC, through an analysis of the irreversibly lost energy at the body trailing edge. For subsonic flows, similar to those evaluated herein, irreversible losses arise mainly as a result of the viscous dissipation leading to a PER evaluation through equation (7).

$$
\operatorname{PER}=1-\frac{\Phi_{T E}}{D V_{\infty}}
$$

Although intended for analysis at the airframe trailing edge, the analysis of PER can be extended further downstream, providing an additional assessment of the energies available for recovery for wake ingesting configurations. By so doing a tacit link can be made between PER and EWC through a recasting of equation (7) by instead considering the recoverable energy outflow, as shown in equation (8). This new factor is simply a recasting and is equivalent to PER for incompressible flows, it will be referred to here as its complement, i.e. $\left(\mathrm{PER}^{\mathrm{c}}\right)$. This then links the energy-based PER and exergy-based EWC when thermal and compressibility effects are negligible (typical for low-speed subsonic flows), as the formulations become approximately equivalent in this regard.

$$
P E R^{c}=\frac{\dot{E}_{a}+\dot{E}_{v}+\dot{E}_{p}}{D V_{\infty}}=\frac{\text { Mechanical Energy Outflow Rate }}{\text { Drag Power }} \approx E W C
$$

\section{III.Numerical Methodology}

\section{A. Geometrical Model and Mesh considerations}

\section{Inviscid, Laminar and Turbulent Flat Plates}

These analyses were modelled in commercial CFD code, STAR CCM+, in a 2D-structured mesh domain with no-slip wall boundaries $(-)$ representing the flat plate $\left(L_{P}\right)$. The near-wall cells were arranged using a hyperbolic tangent distribution, and a first-layer cell thickness of $0.1 \mathrm{~mm}$ to resolve the flat plate boundary layer.

Figure 2(a) shows the mesh and boundary conditions for the extended flat plate domain. The domain was extended a distance of one flat plate length downstream of the trailing edge (indicated by the symmetry plane), allowing the wake to develop downstream. This was done to enable the PBM to quantify the wake energy loss rates downstream of the 
flat plate. To ensure mesh independence, the grid-convergence index method [20] was used at different mesh refinement levels. The final mesh counts for the original (i.e. without the extended symmetry plane) and extended domains were $4 \times 10^{4}$ and $6 \times 10^{4}$, respectively.

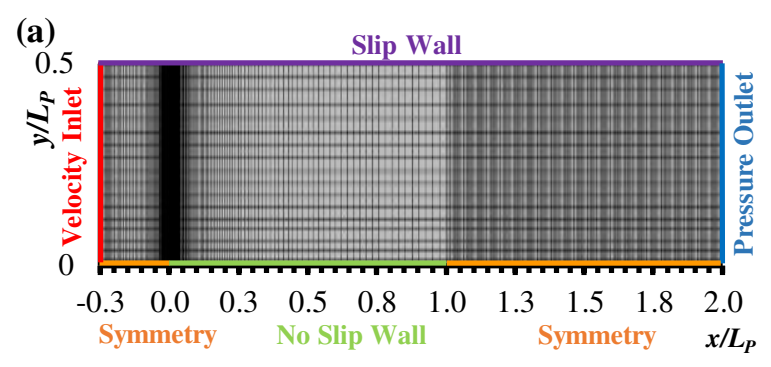

(b)

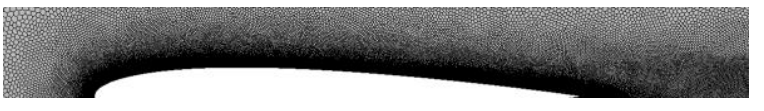

(c)

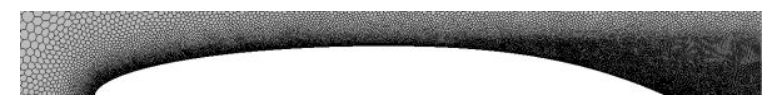

(d)

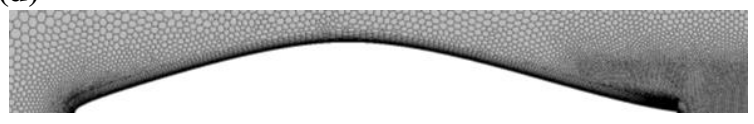

Figure 2 (a) Structured Flat Plate mesh and Unstructured mesh around (b) NACA 0012 Airfoil (c) Myring

Low Drag Body (d) F-57 Low Drag Body

\section{NACA 0012 Airfoil}

The NACA 0012 airfoil was modelled as a 2D body inside a circular computational flow domain, with a diameter of 16 chord lengths and an outer boundary assigned as freestream. The final mesh count for this study was approximately $6.7 \times 10^{5}$ cells created using unstructured polyhedral cells, as shown in Figure 2(b), with 15 prism layer cells used to resolve the boundary layer and yield a wall $\mathrm{y}^{+}$near unity $\left(\mathrm{y}^{+} \approx 1\right)$. The resolution of the mesh was higher in regions where greater computational accuracy was needed, such as the airfoil surface and regions close to the airfoil trailing edge and wake.

\section{Myring Low Drag Body}

The Myring Low Drag Body (MLDB) was modelled in a 2D axisymmetric domain with the flow assumed as steady and incompressible. The domain consisted of a constant velocity inlet four body lengths upstream of the nose, specified to give a Reynolds number equal to the reference cases from literature [21]. The outlet, located nine body lengths 


\section{MUTANGARA ET AL.}

downstream of the trailing edge, was modelled as a pressure boundary set to atmospheric conditions, and the remaining outer boundary as a symmetry plane one body length from the axis line.

The mesh around the body, shown in Figure 2(c), was created using unstructured polyhedral cells with a nondimensional wall distance criterion kept less than unity $\left(\mathrm{y}^{+}<1\right)$ (as required by the $k$ - $\omega$ turbulence model). The final mesh count for this study was approximately $7.5 \times 10^{4}$ cells.

\section{F-57 Low Drag Body}

The F-57 body was modelled using a half section of the 3D axisymmetric body and computational flow domain; this was done to reduce the computational cost by exploiting the flow symmetry. The computational domain consisted of wind tunnel wall dimensions obtained from the experimental investigation by Patel and Lee [22]. Even though the walls were initially of octagonal cross-section, simplification to a square cross-section caused negligible deviation $(<$ $0.5 \%)$ on the force coefficient results [23].

Figure 2(d) shows the mesh around the F-57 body at $\alpha=0^{\circ}$; volumetric refinements were employed to increase the mesh density in regions where viscous effects were prevalent, i.e., around the body and wake regions. The final mesh count obtained after refinement was approximately $5 \times 10^{6}$ cells. For compatibility with the turbulence model used, and to accurately resolve the boundary layer, the maximum wall $\mathrm{y}^{+}$distance was kept less than unity, i.e., $\mathrm{y}^{+}<1$.

\section{B. Boundary Conditions, Turbulence and Transition Models}

\section{Inviscid, Laminar and Turbulent Flat Plates}

The test cases analyzed were modelled as steady, ideal gas flow with a free stream inlet (-) $M_{\infty}=0.2$ and pressure outlet boundaries set to atmospheric conditions. The symmetry boundary condition was used to represent the freestream approaching the flat plate. The length of the plate was given as $L_{P}=2 \mathrm{~m}$ at zero degrees angle of attack $(\alpha$ $=0^{\circ}$ ) yielding a Reynolds number based on the flat plate length of $9 \times 10^{6}$.

The inviscid flow solutions were verified by comparison against an expected drag coefficient solution of zero, which occurs due to the absence of both pressure and skin friction drag. The laminar flow solutions were compared against the theoretical Blasius solution velocity and skin friction coefficient profiles. For turbulent flow, the velocity profiles obtained using the shear-stress transport (SST) $k-\omega$ [24] and Spalart Allmaras turbulence models [25] were compared against the Inner, Logarithmic and Spalding Laws of the wall for verification of the CFD code. 


\section{MUTANGARA ET AL.}

\section{NACA 0012 Airfoil}

The NACA 0012 airfoil boundary conditions were obtained from a numerical study conducted by Jespersen et al. [26]. The flow was assumed to be steady, incompressible and fully turbulent, modelled using the Spalart Allmaras turbulence model [25]. The chord length was given as one meter $(c=1 \mathrm{~m})$, and the airfoil kept at zero degrees angle of attack $\left(\alpha=0^{\circ}\right)$. The freestream $M_{\infty}=0.15$ resulted in a Reynolds number of $6 \times 10^{6}$ based on the airfoil chord.

\section{Myring Low Drag Body}

The MLDB flow was assumed to be steady and incompressible $\left(\operatorname{Re}=10^{7}\right.$ and $\left.M_{\infty}=0.06\right)$, modelled using the $S S T$ $k$ - $\omega$ turbulence model [24] coupled with the $\gamma$ - $\operatorname{Re}_{\theta}$ transition model [27,28] to capture natural flow transition. To affirm the validity of the modelling conditions, the results were compared against numerical and theoretical data with the flow tripped at $\left(x / L_{M}=0.03\right)$, prescribed by Myring [21]. The tripped flow was modelled in STAR-CCM+ using the Turbulence Suppression model, which allowed turbulence effects to be neglected in specified regions.

\section{F-57 Low Drag Body}

The F-57 body flow was assumed to be steady and incompressible $\left(M_{\infty}=0.04\right)$, with a constant velocity inlet condition of $(15.24 \mathrm{~m} / \mathrm{s})$, the body length was given as $(1.219 \mathrm{~m})$ yielding a Reynolds number based on body length of $\left(1.2 \times 10^{6}\right)$ obtained from experimental work by Patel and Lee [22]. The outlet pressure boundary was set to atmospheric conditions with the outer domains modelled as no-slip wall boundaries. The turbulence and transition models were selected as prescribed for aerospace application at low Re. The SST $k$ - $\omega$ turbulence model [24] coupled with the $\gamma$-Re $e_{\theta}$ transition model $[27,28]$ was used. The $\gamma-R e_{\theta}$ transition model was required in order to predict the aerodynamic forces for flow physics involving laminar separation bubbles and flow transition. For validation, the results were compared against experimental work by Patel and Lee [22] with a tripped boundary layer at $x / L_{F} \approx 0.475$.

\section{Comparison with Reference Cases}

For the evaluation of drag over the geometries, two techniques based on momentum analysis introduced in Sections II.A.1 were considered, as well as the power balance method $\left(C D_{\Phi}{ }^{\text {Drela }}\right)$ given in equation (3). To evaluate drag, the more straightforward near-field approach $\left(C D_{n f}\right)$ integrated the stresses over the body surface, whereas the far-field method $\left(C D_{f f}\right)$ relied on momentum-balance across the control-volume outer boundary to obtain the on-body forces. 


\section{A. Inviscid, Laminar and Turbulent Flat Plates}

\section{Inviscid Flat plate}

This section investigates the numerical implementation of the PBM postulated by Drela [9], for the analysis of inviscid flat plates. As a consequence of the flow being inviscid and at an angle of attack of zero degrees, neither pressure nor skin friction drag is present, thus yielding a total drag coefficient result of zero. This was confirmed by all the analysis method solutions being either equal to or approximately zero, as shown in Table 1 at the finest mesh resolution consisting of $2 \times 10^{4}$ cells.

\section{Laminar Flat Plate}

The study was extended to the remainder of the test cases, where the generalised unpowered simplification discussed in Section II.A.3 was used. The volumetric mechanical power and wave energy terms were also additionally dropped as their influence is only expected to be significant for compressible (i.e. $M_{\infty}>0.3$ ) and/or supersonic flows [9,29].

Figure 3(a) shows a comparison between the theoretical Blasius and numerical velocity profiles for the laminar flat plate. Overall, the numerical data showed good agreement with the theoretical, exhibiting small differences $(<0.5 \%)$ at the finest grid refinement level. The small differences observed may be attributed to the fact that Blasius Solution is derived for incompressible flow whereas the test case was modelled as an ideal gas at $M_{\infty}=0.2$, which although minute, does exhibit temperature and density fluctuations.
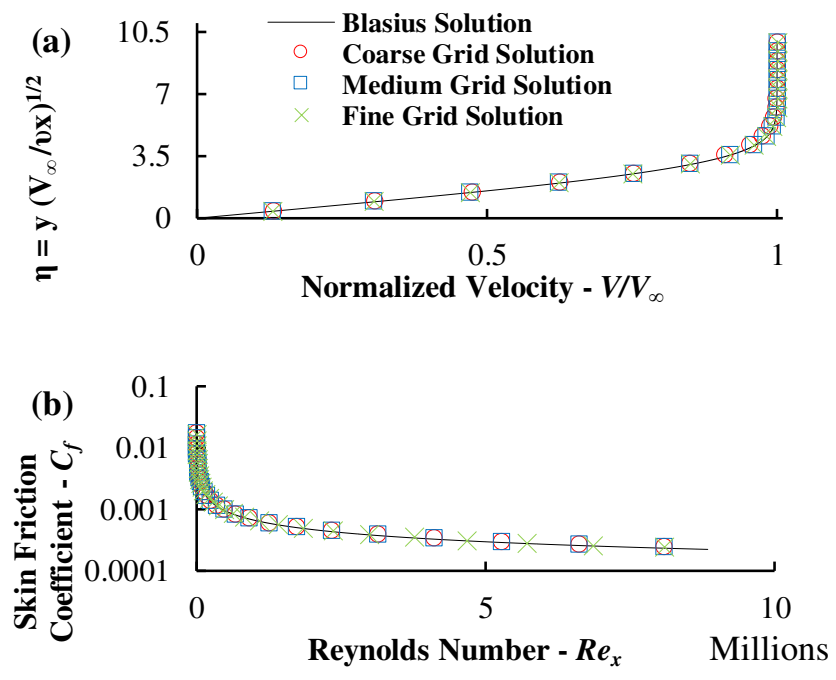

Figure 3 Blasius Solution and Numerical (a) velocity profile (b) Skin friction coefficient comparison at the flat plate trailing edge for various mesh refinement levels 
The numerical skin friction coefficient solutions were monitored and compared against the analytical expression whose profile was obtained from the numerical Blasius solution shown in equation (9).

$$
C_{f} \approx \frac{0.664}{\sqrt{R e_{x}}}
$$

At the finest grid refinement level, the numerical data was seen to closely match the analytical with negligible differences, as shown in Figure 3(b).

Table 1 summarizes the convergence behaviour of the drag coefficient solutions at various mesh refinement levels for the near-field, far-field and power balance methods evaluated at the laminar flat plate trailing edge. An increase in the mesh refinement level was shown to reduce the discrepancies of the far-field, and power balance solutions in comparison to the near-field alternative. A percentage difference of $<2 \%$ was observed between the solutions at the finest mesh refinement level.

\section{Turbulent Flat Plate}

The prior laminar test case was extended to fully turbulent flow analyzed using the SST k- $\omega$ and Spalart Allmaras turbulence models. This analysis focused on observing the behaviour and performance of the PB formulation for turbulent flow regimes. Similar to the laminar flat plate, the numerical solutions were compared against analytical velocity profile distributions. Figure 4 shows this comparison where the numerical, Law of the Wall and Spalding's Law [30] profiles are plotted. The Spalart Allmaras model showed reasonable agreement with the theoretical profiles in both the viscous sublayer and logarithmic regions up to a $\log \mathrm{y}^{+}=2$. The $S S T k$ - $\omega$ model performed similarly well in the viscous sublayer, but further from the wall, in the buffer and logarithmic regions, better agreement was obtained with Spalding's Law $(\kappa=0.4$ and $B=5.5)$. 


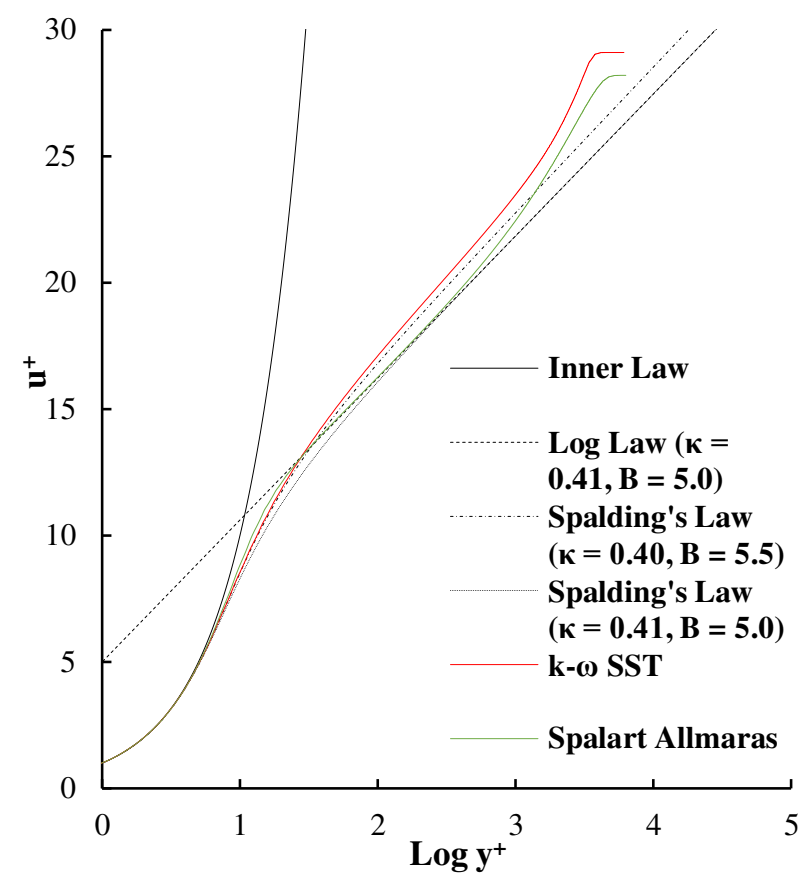

Figure 4 Velocity profile comparison at $x=1.9 m$ against the Law of the Wall and Spalding's Law [30]

Overall, the turbulent flat plate drag coefficients summarized in Table 1 show good agreement with each other with the near-field, far-field and PB solutions all showing differences $<1 \%$.

Table 1 2D Flat Plates in subsonic flow: Near-field, Far-field and Power Balance drag coefficients

\begin{tabular}{cccc}
\hline \multirow{2}{*}{$\begin{array}{c}\text { Mesh } \\
\text { Refinement }\end{array}$} & Near-field & Far-field & $\begin{array}{c}\text { Power } \\
\text { Balance }\end{array}$ \\
\cline { 2 - 4 } & $C D_{n f}$ & $C D_{f f}$ & $C D_{\phi}$ Drela \\
\hline \multirow{2}{*}{ Fine } & \multicolumn{2}{c}{ Inviscid } \\
& \multicolumn{2}{c}{ Laminar } & \\
Coarse & $4.46 \times 10^{-4}$ & $4.57 \times 10^{-4}$ & $4.38 \times 10^{-4}$ \\
Medium & $4.48 \times 10^{-4}$ & $4.50 \times 10^{-4}$ & $4.69 \times 10^{-13}$ \\
Fine & $4.48 \times 10^{-4}$ & $4.50 \times 10^{-4}$ & $4.42 \times 10^{-4}$ \\
& \multicolumn{2}{c}{ Turbulent } & \\
Coarse & $2.71 \times 10^{-3}$ & $2.75 \times 10^{-3}$ & $2.73 \times 10^{-3}$ \\
Medium & $2.77 \times 10^{-3}$ & $2.78 \times 10^{-3}$ & $2.76 \times 10^{-3}$ \\
Fine & $2.77 \times 10^{-3}$ & $2.78 \times 10^{-3}$ & $2.76 \times 10^{-3}$ \\
\hline
\end{tabular}




\section{Turbulence model sensitivity study}

The influence of turbulence modelling on the performance of the PBM was investigated by a comparison of the SST $k-\omega$ and Spalart Allmaras turbulence models. Particular interest was taken in the flow mechanisms which helped explain any discrepancies produced by the turbulence models in their performance predictions. The results for the skin friction coefficient distribution, as well as the numerical drag coefficient data over the flat plate, were recorded to highlight the differences observed for each turbulence model.

Figure 5 shows the skin friction coefficient profiles for the turbulence models and laminar flat plate where the flow regime change (laminar to turbulent) resulted in a significant rise in skin friction. The profile differences observed between the SST k- $\omega$ and Spalart Allmaras turbulence models may be attributed to the differences in turbulence model formulations and the respective near-wall treatments [31].

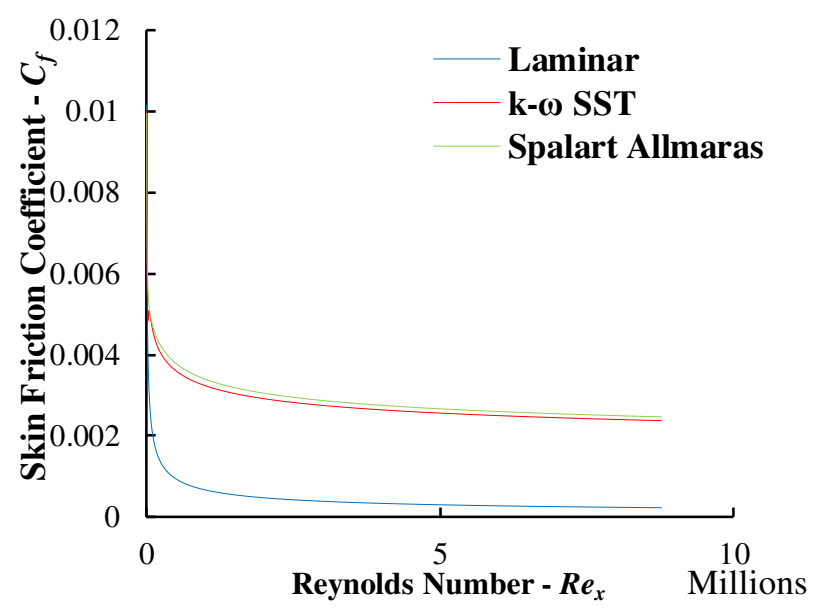

Figure 5 Laminar and Turbulent skin friction coefficient profile comparison over the flat plate

The increased skin friction for the Spalart Allmaras model consequently then lead to the higher total drag coefficient observed in Table 2 . Additionally noted was a drag coefficient difference of approximately $7 \%$ between the turbulence models. Regardless, the far-field and PBM showed negligible difference $(<1 \%)$ when compared against their respective near-field solutions. 
MUTANGARA ET AL.

Table 2 2D Flat Plate: Numerical drag coefficients for SST $k$ - $\omega$ and Spalart Allmaras turbulence models

\begin{tabular}{cccc}
\hline \multirow{2}{*}{$\begin{array}{c}\text { Turbulence } \\
\text { Model }\end{array}$} & Near-field & Far-field & $\begin{array}{c}\text { Power } \\
\text { Balance }\end{array}$ \\
\cline { 2 - 4 } & $C D_{n f}$ & $C D_{f f}$ & $C D_{\Phi}$ Drela \\
SST $k-\omega$ & $2.77 \times 10^{-3}$ & $2.78 \times 10^{-3}$ & $2.76 \times 10^{-3}$ \\
Spalart & $2.96 \times 10^{-3}$ & $2.97 \times 10^{-3}$ & $2.95 \times 10^{-3}$ \\
Allmaras & & & \\
\hline
\end{tabular}

\section{B. NACA 0012 Airfoil}

The NACA 0012 airfoil numerical pressure coefficient profile was compared against experimental data provided by Gregory and O'Reilly [32], and Ladson [33] obtained at $R e=3 \times 10^{6}$ and $6 \times 10^{6}$, respectively. The numerical results from the current study in Figure 6(a), showed a similar general trend with the experimental and good agreement with the numerical data provided by Jespersen et al. [26]. Also shown, in Figure 6(b), is the skin friction coefficient profile, which again showed close agreement with the data provided.
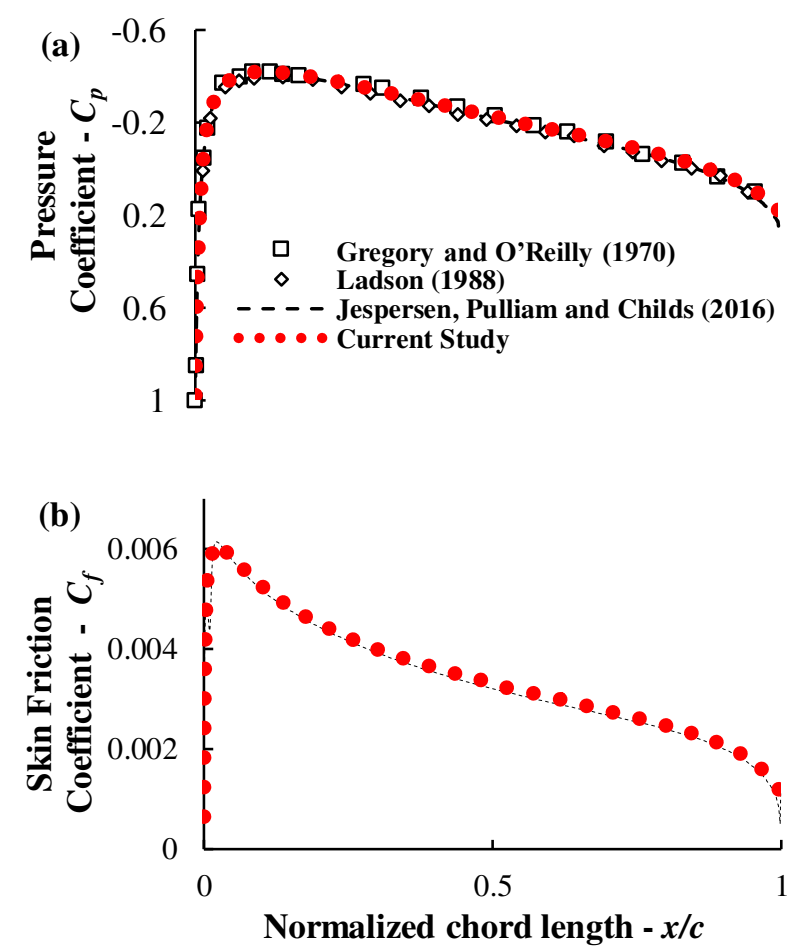

Figure 6 (a)Pressure and (b) Skin Friction Coefficient data for NACA 0012 airfoil at $R e=6 \times 10^{6}$ and Gregory and $O^{\prime}$ Reilly data [32] at $R e=3 \times 10^{6}$ 


\section{Myring Low Drag Body}

The numerical velocity distribution for the current study was compared against the analytical solution provided by Myring [21], showing good agreement with negligible differences between the distributions in Figure 7. In comparison to the tripped flow scenario, the naturally transitioning flow resulted in the fluid accelerating further downstream before transition due to the positive velocity gradient, which extended up to approximately $70 \%$ of the body length. This yielded a natural transition location at $x / L_{M} \approx 0.48$, further aft of the predicted, at $x / L_{M}=0.03$. The validity of these results was confirmed by their agreement with a similar study conducted by Smith et al. [23].

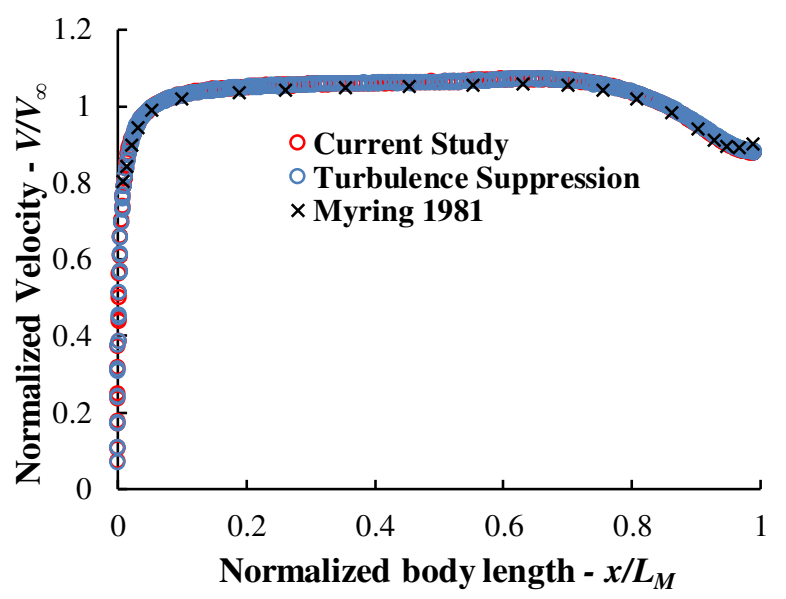

Figure 7 Normalised velocity as a function of Normalised body length for Myring Low Drag Body at $R e=10^{7}$

\section{F-57 Low Drag Body}

Figure 8(a) shows the numerically generated pressure coefficient $\left(C_{\mathrm{p}}\right)$ curve over the F-57 body in the normalised axial direction, i.e., $x / L_{F}$, together with the experimental results of Patel and Lee [22]. The numerical data showed good agreement with the experimental data, with negligible $C_{\mathrm{p}}$ differences at the pressure probed experimental data points. 

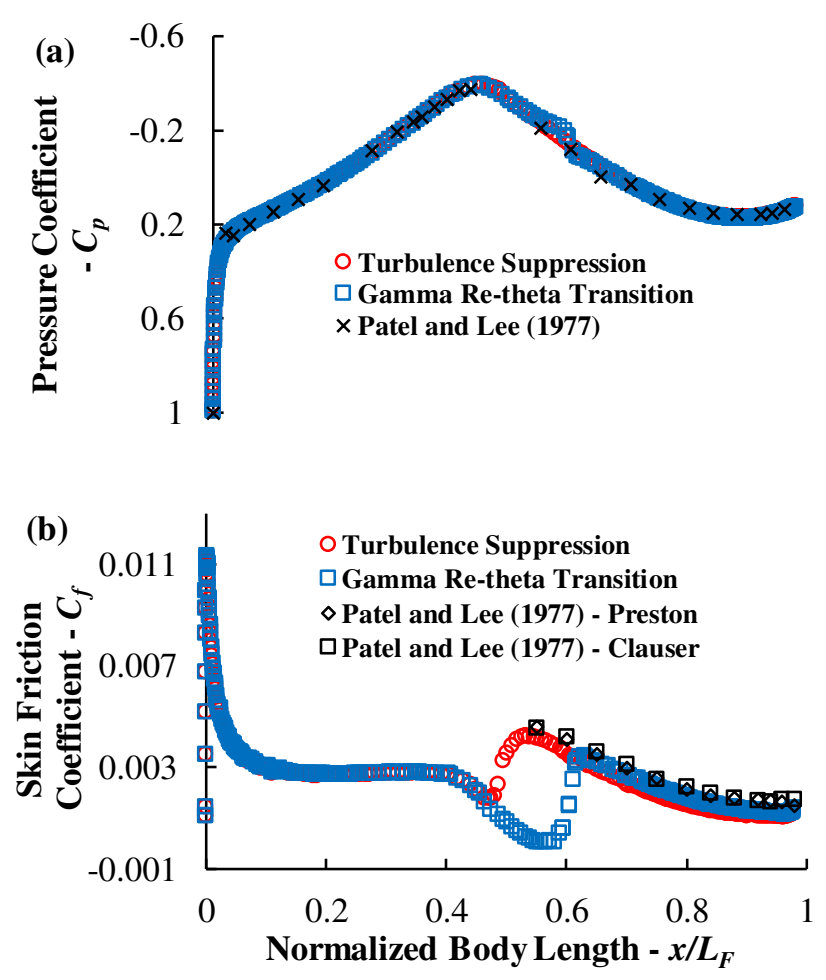

\section{Figure 8 F-57 Low Drag Body (a) Pressure Coefficient and (b) Skin friction Coefficient as a function of}

\section{Normalised body length at $R e=1.2 \times 10^{6}$}

The pressure distribution around the body using the $\gamma-\operatorname{Re}_{\theta}$ transition model showed negligible differences in $C_{\mathrm{p}}$ compared to the Turbulence Suppression (TS) model from $0 \leq x / L_{F} \leq 0.475$. Patel and Lee [22] predicted this region as laminar flow before transition. As natural flow transition is allowed by the $\gamma-R e_{\theta}$ transition model, a separation bubble was observed at $x / L_{F} \approx 0.59$ indicated by the numerical departure in $C_{\mathrm{p}}$ at this location, which is in agreement with a similar study conducted by Smith et al. [23]. The numerical results return to showing negligible deviation in the distribution from $x / L_{F} \approx 0.69$.

Figure 8(b) shows the skin friction coefficient over the body obtained by Patel and Lee [22] using Preston tubes; this method assumed the general Law of the Wall would remain valid for determining the wall shear stress over the body. An alternative approach using the so-called Clauser-plot technique modified using the extended Law of the Wall [34] is also shown. This method was used to correct the Preston tube data to determine the wall shear stress compatible with the wall. 
The TS and $\gamma-R e_{\theta}$ transition data showed the same general trend as the experimental but tended to underpredict the magnitude of skin friction. This may likely have been due to the underprediction of turbulence anisotropy within the boundary layer, which is known to occur with the SST $k$ - $\omega$ turbulence model [31].

The $\gamma-R e_{\theta}$ transition data showed a higher (11\% on average) skin friction distribution in comparison with the TS data for regions $x / L_{F}>0.65$. The likely cause of this requires further investigation but may be attributed to the modification of the boundary layer profile due to natural flow transition, which in contrast to the tripped flow case, allows the fluid to develop further downstream. The changes in the boundary layer profile result in the modification of the skin friction characteristics.

Figure 9(a-b) show the Wake Pressure Coefficient and Wake Centreline Velocity as a function of Normalised body length. The numerical data from the TS and $\gamma-R e_{\theta}$ transition models in Figure 9(a) generally showed good agreement with the experimental data, except for an outlier located at $x / L_{F}=1.3$.

The Wake Centreline Velocity TS data in Figure 9(b) also showed good agreement with the experimental with a maximum deviation of approximately $5 \%$. The normalised velocity distribution for the $\gamma-R_{\theta}$ transition model, however, showed a much higher difference with a maximum of $12 \%$ at $x / L_{F}=1.4$. The likely cause of this deviation can be attributed to the reduced turbulent kinetic energy (TKE) dissipation due to the flow transitioning further downstream in comparison with the tripped flow case, also mentioned by Smith, Meyer and Spedding [35].

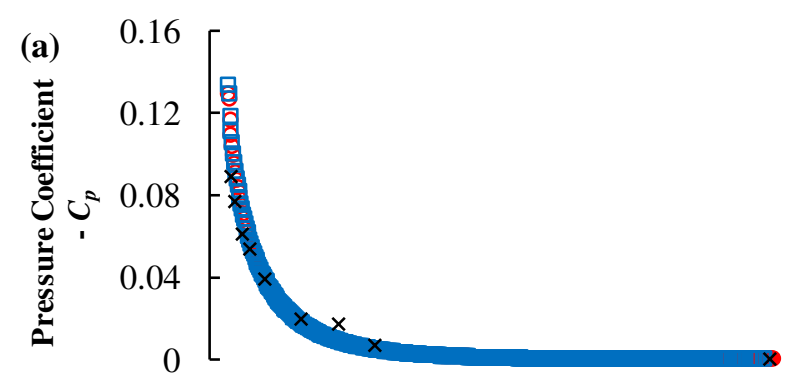




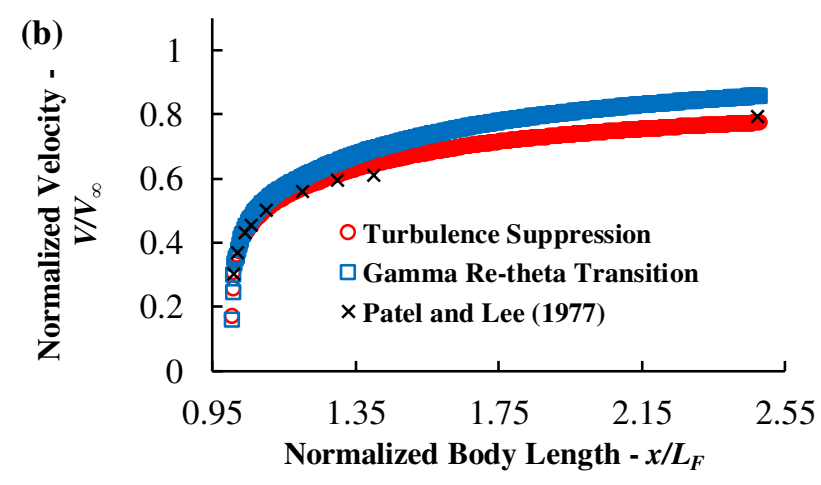

\section{Figure 9 F-57 Low Drag Body (a) Wake Pressure Coefficient (b) Normalized Wake Centreline velocity as a function of the Normalised body length at $R e=1.2 \times 10^{6}$}

\section{V.Unpowered Configuration Wake Energy Analysis}

Drela [9] investigated the dependency of the PB terms on the transverse plane (TP) location. Drela mentions that energy conservation ensures that the PB formulation holds for any Side Cylinder and TP location for a control volume, provided the viscous dissipation $(\Phi)$ is defined to be within the control volume [9]. Similar studies are conducted herein in terms of the non-dimensionalised PB terms to assess the performance of this method when numerically implemented in commercial CFD code STAR CCM+. Of particular interest in this study, was the mechanical energy outflow rate $\dot{\varepsilon}_{m}$, related to the streamwise and transverse kinetic energy deposition rates ( $\dot{E}_{a}$ and $\dot{E}_{v}$ respectively) as well as the pressure-defect work rate $\left(\dot{E}_{p}\right)$. The mechanical energy outflow rate here-in represents the amount of mechanical power available for BLI where

$$
\dot{\varepsilon}_{m}=\dot{E}_{a}+\dot{E}_{v}+\dot{E}_{p}
$$

\section{A. Laminar and Turbulent Flat Plates}

\section{Laminar Flat Plate Wake Energy Analyses at $M_{\infty}=0.20$}

In order to quantify the energy loss rates within the wake, an analysis was conducted with a specific focus on the influence of the Transverse Plane (TP) location on the wake energy. Figure 10(a) shows the wake energy variation of the power balance terms as a function of the TP location. The TP was moved downstream of the flat-plate trailing edge a total distance of $L_{P}=2 \mathrm{~m}$. The far-field and power balance solutions are reported in Table 6 with their solutions given by their respective mean values and standard deviation, shown in brackets. The drag coefficients evaluated using 
the far-field and power balance methods remained relatively constant as the TP progressively moved downstream, demonstrated by the small standard deviations reported in Table 6 . This is in keeping with energy conservation, as explained by Drela [9]. Overall, the solutions showed negligible differences $(<2 \%)$ when compared to the near-field solution.

Table 3 Extended domain Laminar Flat Plate numerical drag coefficient results

\begin{tabular}{cccc}
\hline \hline \multirow{2}{*}{$\begin{array}{c}\text { Mesh } \\
\text { Refinement }\end{array}$} & Near-field & Far-field & $\begin{array}{c}\text { Power } \\
\text { Balance }\end{array}$ \\
\cline { 2 - 4 } & $C D_{n f}$ & $C D_{f f}$ & $C D_{\Phi}$ Drela \\
Fine & $4.50 \times 10^{-4}$ & $\begin{array}{c}4.51 \times 10^{-4} \\
\left( \pm 1.03 \times 10^{-7}\right)\end{array}$ & $\begin{array}{c}4.44 \times 10^{-4} \\
\left( \pm 1.38 \times 10^{-7}\right)\end{array}$ \\
\hline
\end{tabular}

Additionally, the recoverable energy within the wake was found using the PER ${ }^{\mathrm{c}}$ factor discussed in Section II.A.4 through equation (8). Figure 10(a) shows the non-dimensionalised contributions of the Power Balance terms to the mechanical energy (denoted as $\left.\dot{\varepsilon}_{m}\right)$ where the streamwise component $\left(C \dot{E}_{a}\right)$ showed the largest contribution. As the flat plate has no pressure gradient or curvature; contributions of $\dot{E}_{p}$ and $\dot{E}_{v}$ become negligible leading to the flow being dominated by streamwise flow. Figure 10(b) shows the PER ${ }^{\mathrm{c}} \%$ as a function of the TP location. Maximum recovery potential of $\approx 21 \%$ was observed at the flat plate trailing edge, matching analytical solutions provided by Drela and $\mathrm{Lv}$ $[9,36]$. This recovery potential decreased downstream of the flat plate as the recoverable energy viscously dissipated.

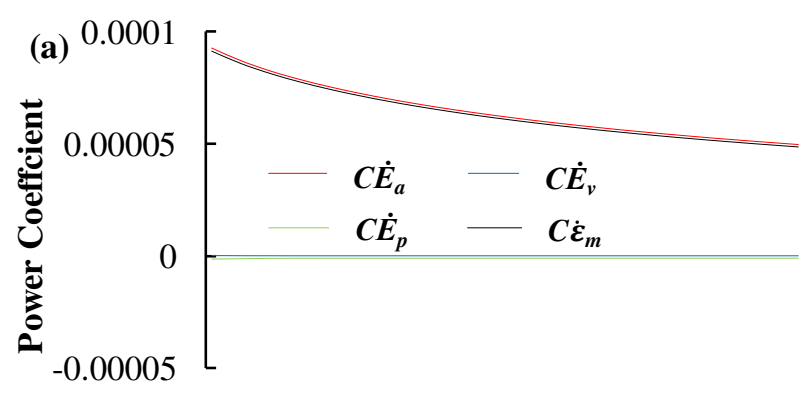




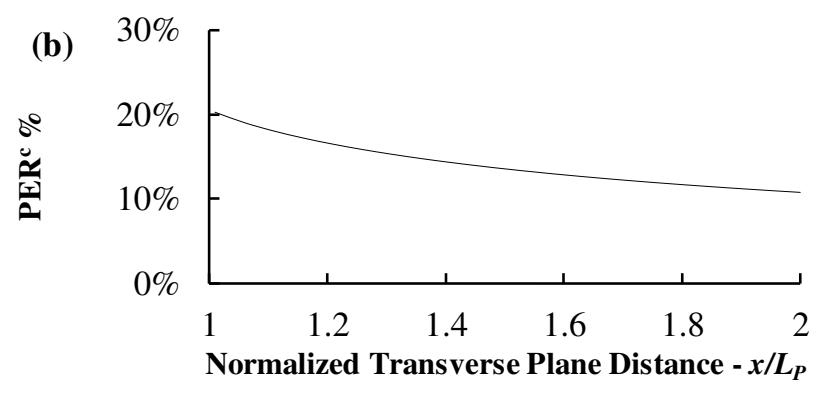

\section{Figure 10 Laminar Flat Plate (a) Decomposition of the mechanical energy outflow rate (b) Recoverable energy in the wake at $R e=9 \times 10^{6}$}

\section{Turbulent Flat Plate Wake Energy Analyses at $M_{\infty}=0.20$}

For the turbulent flat plate, a wake energy analysis was conducted using the SST $k-\omega$ model. Additionally, a turbulence model sensitivity study was done considering the performance of the Spalart Allmaras model commonly used in aerospace applications.

The wake energy analysis in Figure 11(a), showed similar trends to those found in the laminar case study with the power sinks contributing highly to the total drag coefficient coming from the wake streamwise kinetic energy deposition rate and viscous dissipation. The far-field and power balance method solutions reported in Table 4 again remained relatively constant when analyzed downstream of the trailing edge, showing good agreement with the near field solution with a percentage difference of $<1 \%$.

Table 4 Extended domain Turbulent Flat Plate numerical drag coefficient results

\begin{tabular}{cccc}
\hline \multirow{2}{*}{$\begin{array}{c}\text { Mesh } \\
\text { Refinement }\end{array}$} & Near-field & Far-field & $\begin{array}{c}\text { Power } \\
\text { Balance }\end{array}$ \\
\cline { 2 - 4 } & $C D_{n f}$ & $C D_{f f}$ & $C D_{\Phi}$ Drela \\
Fine & $2.78 \times 10^{-3}$ & $\begin{array}{c}2.78 \times 10^{-3} \\
\left( \pm 2.9 \times 10^{-6}\right)\end{array}$ & $\begin{array}{c}2.76 \times 10^{-3} \\
\left( \pm 6.8 \times 10^{-7}\right)\end{array}$ \\
\hline
\end{tabular}

The most significant recoverable energy contribution was observed again to come from the streamwise kinetic energy deposition rate, as shown in Figure 11(a). In this instance, a maximum PER $\%$ of $9 \%$ was observed at the trailing edge decreasing downstream to a value of $3 \%$ at $x / L_{P}=2$, as shown in Figure 11(b). 
MUTANGARA ET AL.
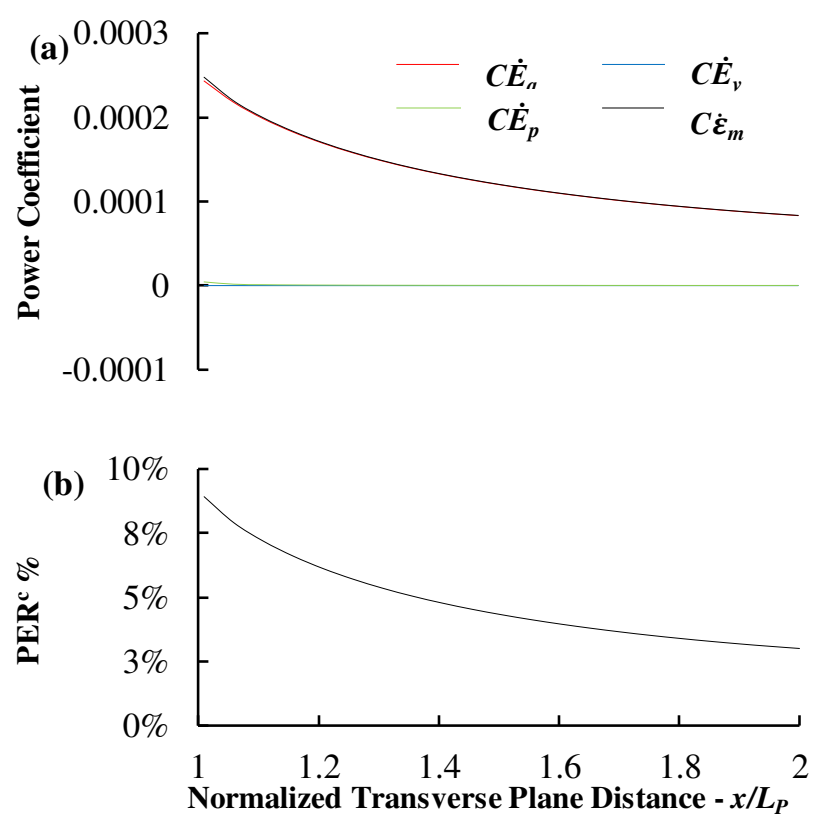

\section{Figure 11 Turbulent Flat Plate (a) Decomposition of the mechanical energy outflow rate (b) Recoverable energy in the wake at $R e=9 \times 10^{6}$}

As the laminar and turbulent case studies were analyzed using the same mesh and boundary conditions, inferences made from their solutions help to highlight the influence of the flow regime on wake energy. On comparing the two flowfields, an essential aspect is realised, i.e. the effects of turbulence on the recoverable wake energy.

Looking at Figure 10 and Figure 11, it can be seen that the amount of recoverable energy for laminar flow is considerably higher than that of the turbulent flow. The main reason for this being the higher viscous dissipation caused by the rise in skin friction for the turbulent flow in Figure 5. The increased skin friction occurred as a result of the steeper velocity gradients and higher shear stresses near the wall of the turbulent flat plate. The higher viscous dissipation contribution to the overall drag reduced the amount of recoverable energy in the wake, seen by the decreased PER $\%$. Similarly, this can also be observed via an analysis of the velocity profiles of the different flow regimes, depicted in Figure 12(b). In the absolute reference frame shown in Figure 12(a) the flat plate moves through a stationary fluid where the area under its velocity profile represents the amount of kinetic energy imparted to a priorly stationary fluid. On comparing the laminar and turbulent profiles in Figure 12(b) it can be observed that the area under the turbulent profile is smaller than that of the laminar. This signifies that for the turbulent profile, the amount of kinetic energy imparted to the flow reduces instead being viscously dissipated as heat and lost irreversibly to the surroundings. 
(a)

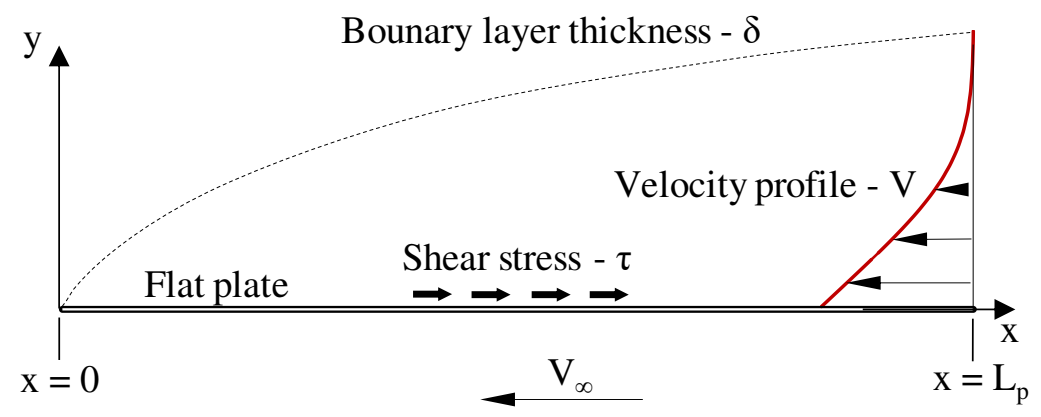

Flat Plate motion

(b)

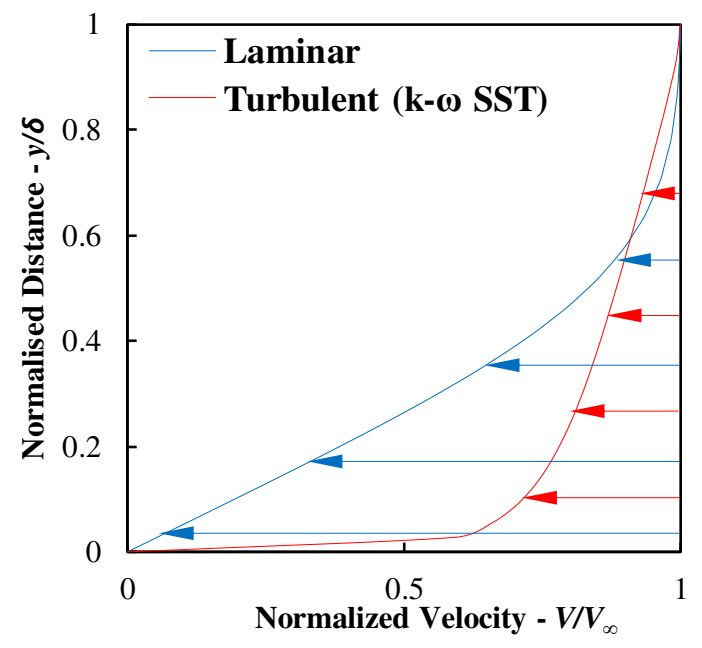

Figure 12 Flat plate (a) Absolute reference frame representation and (b) velocity profile comparison at $x=$

\section{$1.9 \mathrm{~m}$ for laminar and turbulent flow.}

As turbulent flow facilitates the transfer of mass, momentum and energy within the boundary layer; this increases the shear stresses near the wall leading to an increase in the drag coefficient when compared against the laminar flow case. Figure 13(a) shows the effect of the increased energy transfer within the boundary layer, which substantially increases the viscous dissipation.

On comparison of the SST k- $\omega$ and Spalart Allmaras turbulence models, reasonable agreement was observed between the wake streamwise energy profiles shown in Figure 13(a). The viscous dissipation obtained using the Spalart Allmaras model, however, showed a noticeably higher distribution. The agreement between the wake streamwise 
energy profiles allowed for the higher skin friction drag observed for the Spalart Allmaras model to be loosely related to the viscous dissipation quantity. This was somewhat expected as one of the main differences between the turbulence models is their evaluation of turbulent viscosity, which plays a crucial role in the calculation of viscous dissipation. However, on comparison of the component contributions of the PB terms normalised by the drag power depicted in Figure 13(b), no significant differences between the turbulence model solutions were observed. This highlighted that for this study, quantities such as PER and $\mathrm{PER}^{\mathrm{c}}$ could be adequately evaluated using either turbulence model without any significant differences in the solution outcomes.

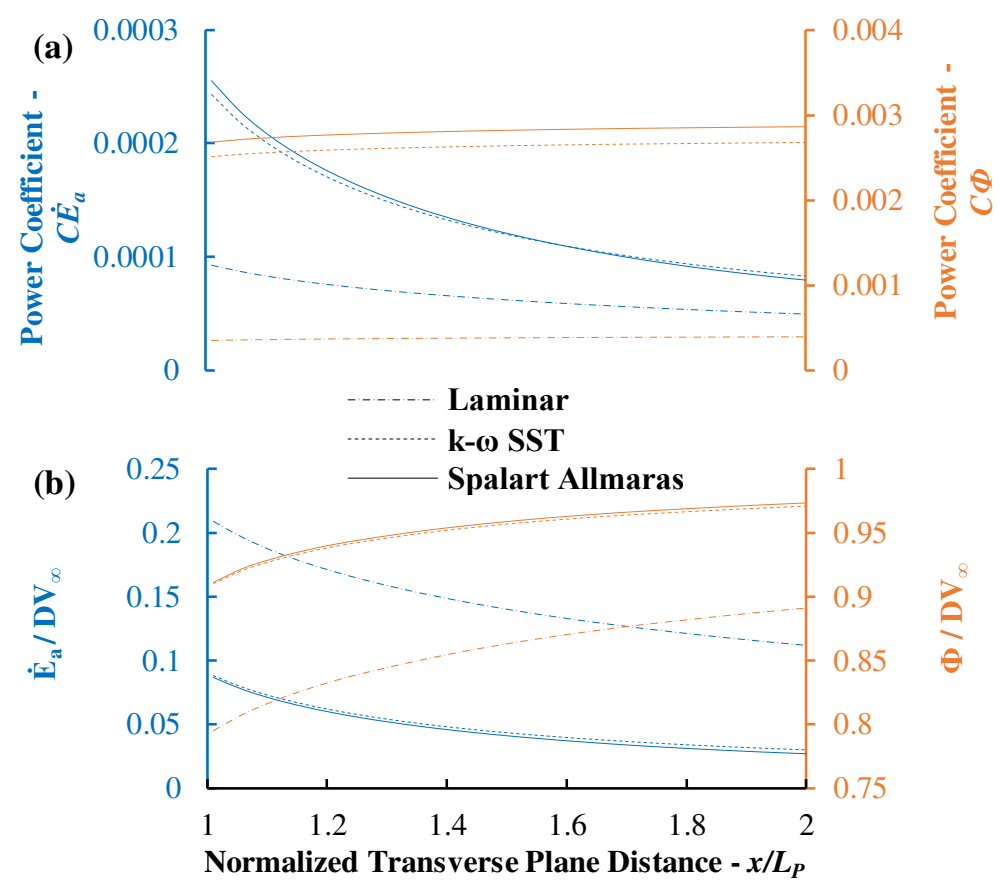

Figure 13 Turbulence model comparison of (a) Axial wake kinetic energy and Viscous Dissipation coefficients and (b) Drag Power normalised Axial wake kinetic energy and Viscous Dissipation rates

\section{B. NACA 0012 Airfoil}

Figure 14(a) shows the wake energy analysis of a NACA 0012 airfoil. The mechanical energy outflow is seen to decay downstream of the body with the lost energy being viscously dissipated as heat. After one body length downstream, the mechanical energy outflow reduces to the streamwise kinetic power as the contributions of the transverse and pressure-defect work rate decrease to zero. 
The mechanical energy lost through the wake was quantified using the Potential for Energy Recovery coefficients as done prior. Figure 14(b) shows the PER ${ }^{c} \%$ as a function of the TP location; a maximum PER $\%$ of $12 \%$ was observed at the trailing edge with this value decaying downstream as the energy dissipated, yielding a value of $4 \%$ at $x / c=2$.
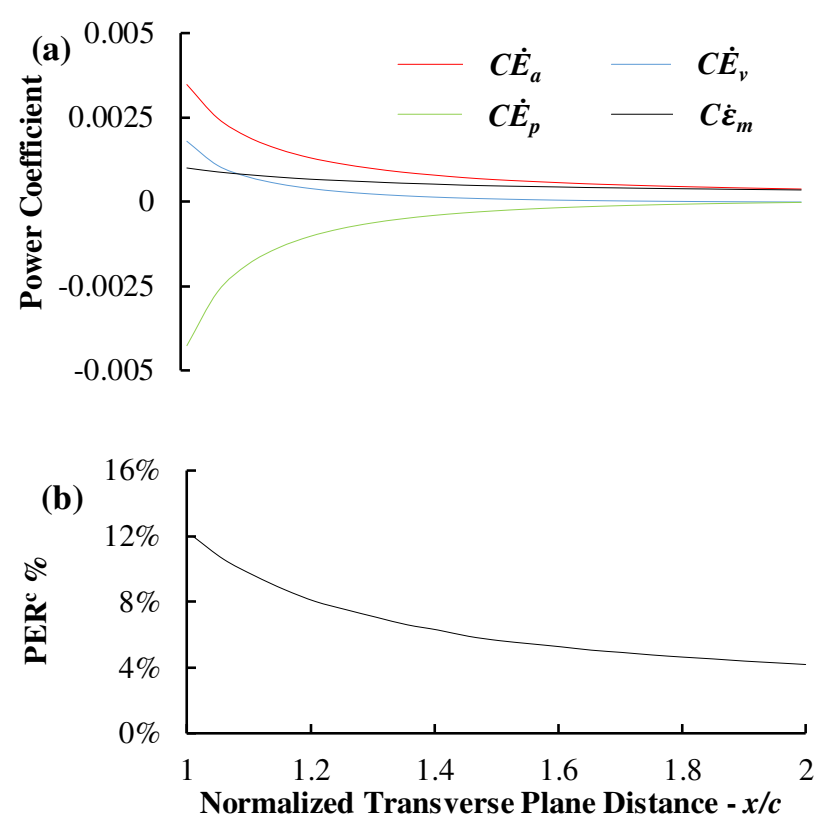

Figure 14 NACA 0012 airfoil (a) Decomposition of the mechanical energy outflow rate (b) Recoverable energy in the wake at $R e=6 \times 10^{6}$

The far-field and power balance solutions in Table 5 were obtained at a TP located one body length downstream (i.e. $x / c=2)$ where the solutions showed negligible difference $(<1 \%)$ when compared against the near-field solution at the finest mesh refinement level.

Table 5 2D NACA 0012 Airfoil: Near-field, far-field and power balance method drag coefficients

\begin{tabular}{cccc}
\hline \multirow{2}{*}{$\begin{array}{c}\text { Mesh } \\
\text { Refinement }\end{array}$} & Near-field & Far-field & $\begin{array}{c}\text { Power } \\
\text { Balance }\end{array}$ \\
\cline { 2 - 4 } & $C D_{n f}$ & $C D_{f f}$ & $C D_{\Phi}$ Drela \\
Coarse & $8.43 \times 10^{-3}$ & $8.47 \times 10^{-3}$ & $7.96 \times 10^{-3}$ \\
Medium & $8.36 \times 10^{-3}$ & $8.36 \times 10^{-3}$ & $8.18 \times 10^{-3}$ \\
Fine & $8.34 \times 10^{-3}$ & $8.33 \times 10^{-3}$ & $8.36 \times 10^{-3}$ \\
\hline \hline
\end{tabular}




\section{Myring Low Drag Body}

Figure 15(a) shows the MLDB wake energy analysis; the overall behaviour of the terms is comparable to the NACA 0012 airfoil shown in Figure 14(a). Downstream of the trailing edge, the mechanical energy outflow reduces to the streamwise kinetic energy as contributions from the transverse and pressure-defect work rate decrease to zero, also shown in Figure 15(a). Figure 15(b), provides the estimated PER $\%$, which was observed to have a value of $11 \%$ at the body trailing end, gradually decaying downstream to approximately $5 \%$ at $x / L_{M}=2$.
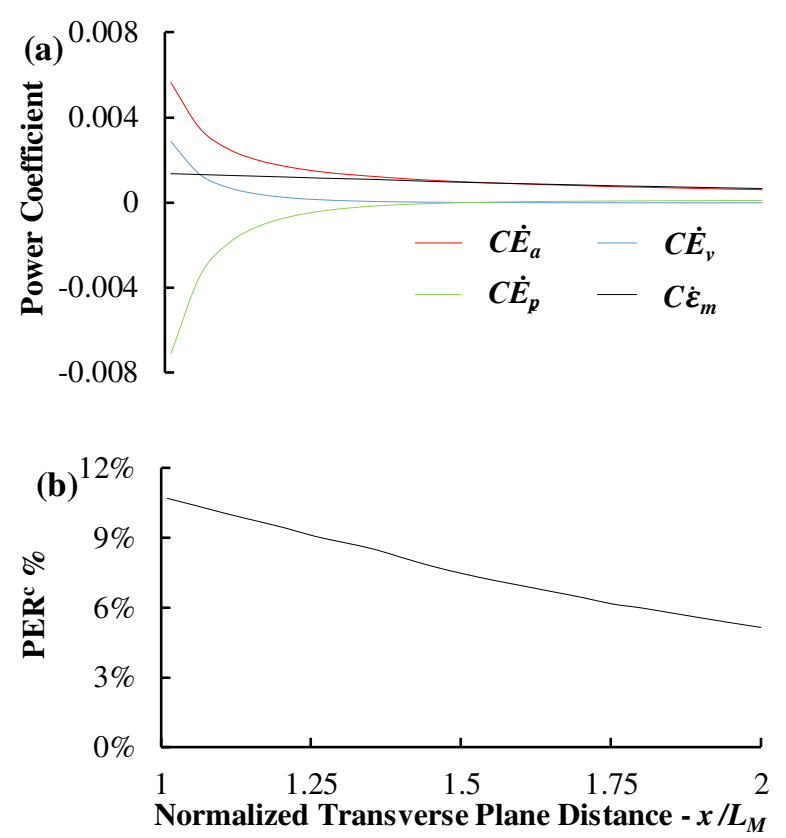

Figure 15 Myring Low Drag Body (a) Decomposition of the mechanical energy outflow rate (b) Recoverable energy in the wake at $R e=10^{7}$

The drag coefficient results reported in Table 6 are non-dimensionalised in terms of body volume $(V)$, which is typical for bodies of revolution (BoR). The drag coefficients for BoR in this and sections to follow will be nondimensionalised using equations (11).

$$
C D=\frac{D}{\frac{1}{2} \rho_{\infty} V_{\infty}^{2} A_{\text {ref }}}\left(\frac{A_{\text {ref }}}{V^{\frac{2}{3}}}\right) ; C \dot{E}_{a}=\frac{\dot{E}_{a}}{\frac{1}{2} \rho_{\infty} V_{\infty}^{3} A_{r e f}}\left(\frac{A_{r e f}}{V^{\frac{2}{3}}}\right)
$$


MUTANGARA ET AL.

Table 6 summarizes the drag coefficient solutions for the near-field, far-field and power balance method. The far-field and power balance solutions were obtained at a TP located one body length downstream of the MLDB; where their solutions were shown to be within $<1 \%$ of the near-field value.

Table 6 Axisymmetric MLDB: Near-field, far-field and power balance method volume-based drag coefficients

\begin{tabular}{cccc}
\hline \multirow{2}{*}{$\begin{array}{c}\text { Mesh } \\
\text { Refinement }\end{array}$} & Near-field & Far-field & $\begin{array}{c}\text { Power } \\
\text { Balance }\end{array}$ \\
\cline { 2 - 4 } & $C D_{n f}$ & $C D_{f f}$ & $C D_{\Phi}$ Drela \\
\hline Coarse & $1.78 \times 10^{-2}$ & $1.83 \times 10^{-2}$ & $1.66 \times 10^{-2}$ \\
Medium & $1.26 \times 10^{-2}$ & $1.27 \times 10^{-2}$ & $1.25 \times 10^{-2}$ \\
Fine & $1.26 \times 10^{-2}$ & $1.27 \times 10^{-2}$ & $1.26 \times 10^{-2}$ \\
\hline \hline
\end{tabular}

\section{F-57 Low Drag Body}

Figure 16(a) shows the wake energy analysis for the F-57 Low Drag Body, the trends observed in prior analyses for the NACA 0012 airfoil and MLDB w.r.t the mechanical energy are also seen here. The mechanical energy distribution is shown to decay gradually downstream until it matches the streamwise kinetic energy, which then becomes the main contributor to the overall mechanical energy. Figure 16(b) shows the wake energy available for recovery, a maximum $\operatorname{PER}^{\mathrm{c}} \%$ of $12 \%$ was observed at the body trailing end decaying downstream to approximately $5 \%$ at $x / L_{F}=2$.
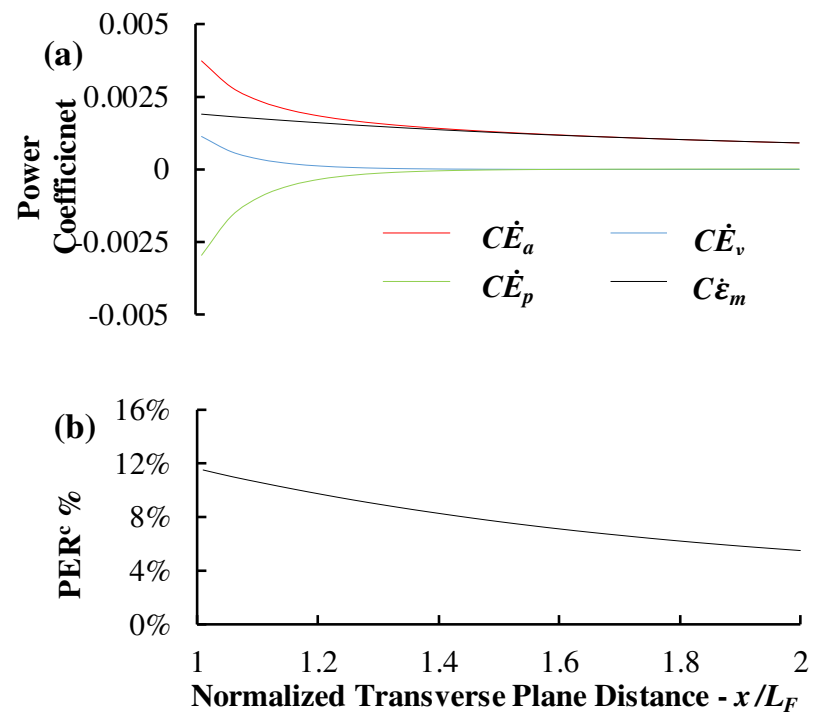

Figure 16 (a) Decomposition of the mechanical energy outflow rate (b) F-57 Low Drag Body Recoverable energy in the wake at $R e=1.2 \times 10^{6}$ 
MUTANGARA ET AL.

The drag over the body was analyzed using the far-field and power balance methods and compared to the near-field solution as done prior. At a TP located one body length downstream; the solutions from the far-field and power balance methods were seen to show agreement with the near-field solution to within $<2 \%$ as shown in Table 7 .

Table 7 F-57 Low Drag Body: Near-field, far-field and power balance volume-based drag coefficients

\begin{tabular}{cccc}
\hline \multirow{2}{*}{$\begin{array}{c}\text { Mesh } \\
\text { Refinement }\end{array}$} & Near-field & Far-field & $\begin{array}{c}\text { Power } \\
\text { Balance }\end{array}$ \\
\cline { 2 - 4 } & $C D_{n f}$ & $C D_{f f}$ & $C D_{\Phi}$ Drela \\
\hline Coarse & $1.66 \times 10^{-2}$ & $1.66 \times 10^{-2}$ & $1.59 \times 10^{-2}$ \\
Medium & $1.65 \times 10^{-2}$ & $1.66 \times 10^{-2}$ & $1.61 \times 10^{-2}$ \\
Fine & $1.65 \times 10^{-2}$ & $1.66 \times 10^{-2}$ & $1.62 \times 10^{-2}$ \\
Very Fine & $1.65 \times 10^{-2}$ & $1.66 \times 10^{-2}$ & $1.62 \times 10^{-2}$ \\
\hline
\end{tabular}

\section{VI.Discussion of Results}

The $\mathrm{PER}^{\mathrm{c}}$ performance of the various bodies was compared using the solutions obtained in the prior sections. The laminar flat plate results, shown in Figure 17(a), provided an upper limit for PER $^{\mathrm{c}}$ with a value of $21 \%$ based on Blasius solution, whereas the turbulent flat plate gave the lower limit of $9 \%$. The decrease in $\mathrm{PER}^{\mathrm{c}}$ for the turbulent flat plate occurred as a result of the increased viscous losses caused by the higher shear stresses near the flat plate wall. The effect of increasing Reynolds number yielded a similar outcome, with less kinetic energy being imparted to the flow and instead being viscously dissipated as heat to the surroundings. This was clearly shown on comparison of the fully turbulent NACA 0012 airfoil and flat plate, where the decrease in PER ${ }^{\mathrm{c}}$ observed for the higher Reynolds number turbulent flat plate was accompanied by an increase in viscous dissipation as depicted in Figure 17(b). 

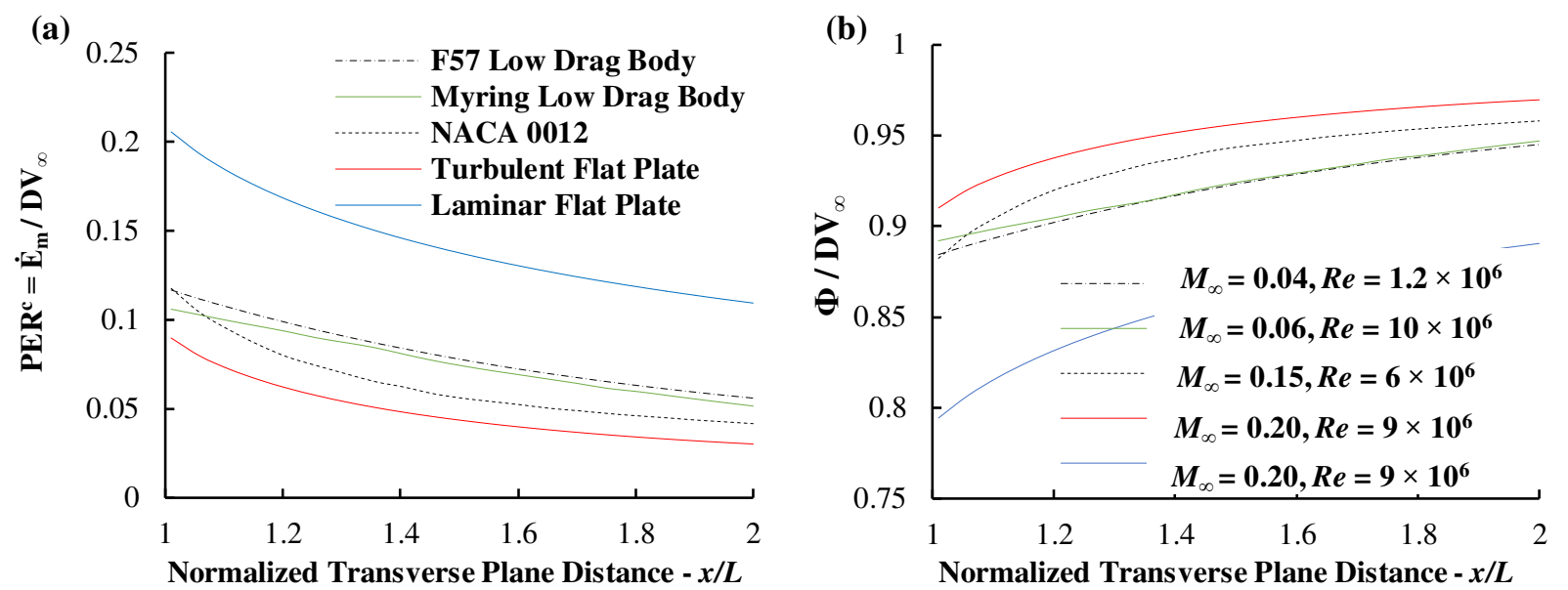

Figure 17 (a) PER $^{\mathrm{c}}$ and (b) Drag Power normalised Viscous Dissipation rate comparison at various Reynolds

\section{and Mach numbers}

From these observations, it would be expected that the MLDB, being at the highest Reynolds number of $10^{7}$, would yield the lowest $\mathrm{PER}^{\mathrm{c}}$ and highest viscous dissipation component relative to its drag power. However, on analysis, a $\mathrm{PER}^{\mathrm{c}}$ of $11 \%$ was obtained, much higher than expected for this Reynolds number. This occurred as a result of the body having significant regions of natural laminar flow with a transition location at $x / L_{M} \approx 0.48$. As a result, the benefits of natural laminar flow could be realised, resulting in a $\mathrm{PER}^{\mathrm{c}}$ and viscous dissipation component comparable to that of the lowest Reynolds number F57 case study, as shown in Figure 17(a) and (b).

Although the F57 Low Drag Body was analyzed at the most favourable Reynolds number with regards to PER ${ }^{\mathrm{c}}$, its aftbody flow was not desirable. The strong pressure gradient and laminar separation bubble observed in this region resulted in lower energy recovery potential at its trailing edge. Therefore from this, it is opined that in order to increase a body's energy recovery potential, careful consideration must be taken with regards to the surface curvature, focusing mainly on utilizing the benefits of natural laminar flow while avoiding strong pressure gradients and separation.

\section{VII.Conclusions}

The PBM decomposition of the various mechanisms contributing towards airframe drag provided a more in-depth description compared to the usual sum of pressure and skin friction drag components. This allowed for a unique 
MUTANGARA ET AL.

grouping of components into non-recoverable and recoverable energy sources, which becomes of particular interest for BLI.

The flat plate case studies identified the differences between laminar and turbulent flow w.r.t the amount of recoverable energy available for BLI. The laminar flat plate showed a higher energy recovery potential for BLI (i.e. $\operatorname{PER}^{\mathrm{c}} \%=21 \%$ ) in comparison to turbulent flow (i.e. $\mathrm{PER}^{\mathrm{c}} \%=9 \%$ ). This was observed due to the fact that laminar flow loses less energy irreversibly to the surroundings, following from the reduced influence of skin friction.

The turbulent flat plate case studies also compared the Spalart Allmaras and SST $k$ - $\omega$ turbulence models w.r.t their drag coefficients using near-field, far-field, and power balance methods. The study showed that even though the two turbulence model drag coefficients differed by approximately 7\%, the drag prediction performance of the power balance and far-field methods were unaffected, with their results still showing good agreement with their respective near-field solutions.

The final case studies focused on numerically verifying the PBM drag decompositions on a 2D NACA 0012 airfoil at $R e=6 \times 10^{6}$, a $2 \mathrm{D}$ axisymmetric body of revolution (MLDB) at $R e=10^{7}$ and a $3 \mathrm{D} \mathrm{BoR}$ with a strong pressure gradient at $R e=1.2 \times 10^{6}$. The analyses consistently showed that the results for all three methods (near-field, far-field and power balance) were in close agreement to within $2 \%$, increasing the reliability of the solutions due to the cross-validation procedure. As expected, the bodies all showed maximal recovery potential at their trailing ends, with the NACA 0012 airfoil and F-57 Low Drag Body yielding PER $\%$ s of 12\%, and 11\% for the MLDB. The PER ${ }^{\mathrm{c}}$ performance of the transitional flow MLDB highlighted the importance of surface topology in increasing the recovery potential, specifically by utilizing the benefits of natural laminar flow, which reduces the irreversible losses.

\section{Acknowledgements}

The authors would like to thank the University of Pretoria Department of Research and Innovation as well as Cranfield School of Aerospace, Transport and Manufacturing for their support and funding during the development of this work. 


\section{MUTANGARA ET AL.}

\section{References}

[1] Habermann, A. L., Bijewitz, J., Seitz, A., and Hornung, M. "Performance Bookkeeping for Aircraft Configurations with Fuselage Wake-Filling Propulsion Integration.” CEAS Aeronautical Journal, 2019, pp. 1-23. https://doi.org/10.1007/s13272-019-00434-w.

[2] Giles, M. B., and Cummings, R. M. "Wake Integration for Three-Dimensional Flowfield Computations: Theoretical Development.” Journal of Aircraft, Vol. 36, No. 2, 1999, pp. 357-365. https://doi.org/10.2514/2.2465.

[3] Paparone, L., and Tognaccini, R. "Computational Fluid Dynamics-Based Drag Prediction and Decomposition.” AIAA Journal, Vol. 41, No. 9, 2003, pp. 1647-1657. https://doi.org/10.2514/2.7300.

[4] Mele, B., Ostieri, M., and Tognaccini, R. “Aircraft Lift and Drag Decomposition in Transonic Flows.” Journal of Aircraft, Vol. 54, No. 5, 2017, pp. 1933-1944. https://doi.org/10.2514/1.C034288.

[5] Destarac, D., and Van Der Vooren, J. "Drag/Thrust Analysis of Jet-Propelled Transonic Transport Aircraft; Definition of Physical Drag Components.” Aerospace Science and Technology, Vol. 8, No. 6, 2004, pp. 545-556. https://doi.org/10.1016/j.ast.2004.03.004.

[6] Yamazaki, W., Matsushima, K., and Nakahashi, K. "Drag Prediction, Decomposition and Visualization in Unstructured Mesh CFD Solver of TAS-Code.” International Journal for Numerical Methods in Fluids, Vol. 57, No. 4, 2008, pp. 417436. https://doi.org/10.1002/fld.1643.

[7] Yamazaki, W., Matsushima, K., and Nakahashi, K. “Aerodynamic Design Optimization Using the Drag-Decomposition Method.” AIAA Journal, Vol. 46, No. 5, 2008, pp. 1096-1106. https://doi.org/10.2514/1.30342.

[8] Sanders, D. S., and Laskaridis, P. "Full-Aircraft Energy-Based Force Decomposition Applied to Boundary-Layer Ingestion.” AIAA Journal, Vol. 58, No. 10, 2020, pp. 4357-4373. https://doi.org/10.2514/1.J058695.

[9] Drela, M. "Power Balance in Aerodynamic Flows." AIAA Journal, Vol. 47, No. 7, 2009 , pp. $1761-1771$. https://doi.org/10.2514/1.42409.

[10] Arntz, A. Civil Aircraft Aero-Thermo-Propulsive Performance Assessment by an Exergy Analysis of High-Fidelity CFDRANS Flow Solutions. PhD Dissertation, Lille 1 University - Sciences and Technologies, 2014.

[11] Arntz, A., and Atinault, O. "Exergy-Based Performance Assessment of a Blended Wing-Body with Boundary-Layer Ingestion.” AIAA Journal, Vol. 53, No. 12, 2015, pp. 3766-3776. https://doi.org/10.2514/1.J054072.

[12] Arntz, A., and Hue, D. "Exergy-Based Performance Assessment of the NASA Common Research Model." AIAA Journal, Vol. 54, No. 1, 2016, pp. 88-100. https://doi.org/10.2514/1.J054127.

[13] Aguirre, M. A., and Duplaa, S. "Exergetic Drag Characteristic Curves.” AIAA Journal, Vol. 57, No. 7, 2019, pp. 27462757. https://doi.org/10.2514/1.J057985.

[14] Aguirre, M. A., Duplaa, S., and Carbonneau, X. "2D Flow Field Analysis by the Exergetic Method.” AIAA Aviation 2019 


\section{MUTANGARA ET AL.}

Forum, Jun, 2019, pp. 1-27. https://doi.org/10.2514/6.2019-2925.

[15] Aguirre, M., Duplaa, S., and Carbonneau, X. "Vortex Exergy Prediction." 54th 3AF International Conference on Applied Aerodynamics, Association Aéronautique et Astronautique de France (3AF), Paris, France, 2019, pp. 1-10.

[16] Aguirre, M., Duplaa, S., Carbonneau, X., and Turnbull, A. “A Systematic Analysis of the Mechanical Exergy of an Airfoil by Using Potential Flow , Euler \& RANS.” 24ème Congrès Français de Mécanique, Association Française de Mécanique (AFM), Courbevoie, France, 2019, pp. 1-22.

[17] Arntz, A., Atinault, O., Destarac, D., and Merlen, A. "Exergy-Based Aircraft Aeropropulsive Performance Assessment: CFD Application to Boundary Layer Ingestion.” 32nd AIAA Applied Aerodynamics Conference, AIAA Paper 2014-2573, Jun, 2014, https://doi.org/10.2514/6.2014-2573.

[18] Arvind, R., and Peijian, L. "Conceptual Analysis of Boundary Layer Ingestion towards Propulsion Integration." Proceedings of the International Society of Air Breathing Engines (ISABE), ISABE, Indianapolis, 2013, pp. 1-15.

[19] Smith, L. H. "Wake Ingestion Propulsion Benefit." Journal of Propulsion and Power, Vol. 9, No. 1, 1993 , pp. 74-82. https://doi.org/10.2514/3.11487.

[20] American Society of Mechanical Engineers (ASME). Standard for Verification and Validation in Computational Fluid Dynamics and Heat Transfer. American Society of Mechanical Engineers (ASME), New York, 2009, pp 11-14.

[21] Myring, D. F. A Theoretical Study of the Effects of Body Shape and Mach Number on the Drag of Bodies of Revolution in Subcritical Axisymmetric Flow. Royal Aircraft Establishment, Farnborough, Hants, UK, Technical Report 81005, 1981.

[22] Patel, V. C., and Lee, Y. T. Thick Axisymmetric Turbulent Boundary Layer and Near Wake of a Low-Drag Body of Revolution. Institute of Hydraulic Research University of Iowa, IIHR Report No. 210, 1977.

[23] Smith, L., Craig, K. J., Meyer, J. P., and Spedding, G. R. "Modifying Low-Drag Bodies to Generate Lift: A Computational Study.” Journal of Aircraft, Vol. 54, No. 3, 2017, pp. 1150-1161. https://doi.org/10.2514/1.C034051.

[24] Menter, F. R. “Two-Equation Eddy-Viscosity Turbulence Models for Engineering Applications.” AIAA Journal, Vol. 32, No. 8, 1994, pp. 1598-1605. https://doi.org/10.2514/3.12149.

[25] Spalart, P., and Allmaras, S. "A One-Equation Turbulence Model for Aerodynamic Flows.” 30th Aerospace Sciences Meeting and Exhibit, AIAA Paper 1992-439, Jan, 1992. https://doi.org/10.2514/6.1992-439.

[26] Jespersen, D. C., Pulliam, T. H., and Childs, M. L. OVERFLOW Turbulence Modeling Resource Validation Results. NAS Technical Report: NAS-2016-01, 2016.

[27] Menter, F. R., Langtry, R. B., Likki, S. R., Suzen, Y. B., Huang, P. G., and Völker, S. “A Correlation-Based Transition Model Using Local Variables Part I - Model Formulation.” Proceedings of the ASME Turbo Expo 2004, New York, Vol. 4, 2004, pp. 57-67. https://doi.org/10.1115/GT2004-53452.

[28] Langtry, R. B., Menter, F. R., Likki, S. R., Suzen, Y. B., Huang, P. G., and Völker, S. “A Correlation-Based Transition 


\section{MUTANGARA ET AL.}

Model Using Local Variables - Part II: Test Cases and Industrial Applications.” Journal of Turbomachinery, Vol. 128, No. 3, 2006, pp. 423-434. https://doi.org/10.1115/1.2184353.

[29] Uranga, A., Drela, M., Hall, D. K., and Greitzer, E. M. “Analysis of the Aerodynamic Benefit from Boundary Layer Ingestion for Transport Aircraft.” AIAA Journal, Vol. 56, No. 11, 2018, pp. 4271-4281. https://doi.org/10.2514/1.J056781.

[30] Spalding, D. B. “A Single Formula for the "Law of the Wall.” Journal of Applied Mechanics, Vol. 28, No. 3, 1961, pp. 455-458. https://doi.org/10.1115/1.3641728.

[31] Siemens. Simcenter STAR-CCM+® Documentation. CD-Adapco, Melville, NY, 2019, pp 3657-3834, 7094-7197.

[32] Gregory, N., and O'Reilly, C. L. Low-Speed Aerodynamic Characteristics of NACA 0012 Aerofoil Section. Ministry of Defence Aeronautical Research Council, Reports and Memoranda No. 3726*, 1970.

[33] Ladson, C. L. Effects of Independent Variation of Mach and Reynolds Numbers on the Low-Speed Aerodynamic Characteristics of the NACA 0012 Airfoil Section. NASA Langley Research Center, Report No TM-4074, 1988.

[34] Patel, V. C. "A Unified View of the Law of the Wall Using Mixing-Length Theory.” Aeronautical Quarterly, Vol. 24, No. 1, 1973, pp. 55-70. https://doi.org/10.1017/S0001925900006429.

[35] Smith, L., Davis, T. W., Spedding, G., and Meyer, J. P. "Numerical and Laboratory Experiments on a New Wing-BodyTail Configuration." 54th AIAA Aerospace Sciences Meeting, AIAA Paper 2016-0800, Jan, 2016. https://doi.org/10.2514/6.2016-0800.

[36] Lv, L. Theoretical and Experimental Investigation of Boundary Layer Ingestion for Aircraft Application PhD Thesis. TU Delft University, 2019. 
2021-07-30

Potential for energy recovery of unpowered configurations using power balance method computations

Mutangara, Ngonidzashe E.

AIAA

Mutangara NE, Smith L, Craig KJ, Sanders DS. (2021) Potential for energy recovery of unpowered configurations using power balance method computations. Journal of Aircraft, Volume 58, Issue 6, November 2021, pp.1364-1374

https://doi.org/10.2514/1.C036172

Downloaded from Cranfield Library Services E-Repository 